\title{
TRICHOTOMY, STABILITY, AND OSCILLATION OF A FUZZY DIFFERENCE EQUATION
}

\author{
G. STEFANIDOU AND G. PAPASCHINOPOULOS
}

Received 10 November 2003

We study the trichotomy character, the stability, and the oscillatory behavior of the positive solutions of a fuzzy difference equation.

\section{Introduction}

Difference equations have already been successfully applied in a number of sciences (for a detailed study of the theory of difference equations and their applications, see $[1,2,7$, $8,11]$.

The problem of identifying, modeling, and solving a nonlinear difference equation concerning a real-world phenomenon from experimental input-output data, which is uncertain, incomplete, imprecise, or vague, has been attracting increasing attention in recent years. In addition, nowadays, there is an increasing recognition that for understanding vagueness, a fuzzy approach is required. The effect is the introdution and the study of the fuzzy difference equations (see $[3,4,13,14,15]$ ).

In this paper, we study the trichotomy character, the stability, and the oscillatory behavior of the positive solutions of the fuzzy difference equation

$$
x_{n+1}=A+\frac{\sum_{i=1}^{k} c_{i} x_{n-p_{i}}}{\sum_{j=1}^{m} d_{j} x_{n-q_{j}}},
$$

where $k, m \in\{1,2, \ldots\}, A, c_{i}, d_{j}, i \in\{1,2, \ldots, k\}, j \in\{1,2, \ldots, m\}$, are positive fuzzy numbers, $p_{i}, i \in\{1,2, \ldots, k\}, q_{j}, j \in\{1,2, \ldots, m\}$, are positive integers such that $p_{1}<p_{2}<$ $\cdots<p_{k}, q_{1}<q_{2}<\cdots<q_{m}$, and the initial values $x_{i}, i \in\{-\pi,-\pi+1, \ldots, 0\}$, where

$$
\pi=\max \left\{p_{k}, q_{m}\right\}
$$

are positive fuzzy numbers.

Studying a fuzzy difference equation results concerning the behavior of a related family of systems of parametric ordinary difference equations is required. Some necessary results 
concerning the corresponding family of systems of ordinary difference equations of (1.1) have been proved in [16] and others are given in this paper.

\section{Preliminaries}

We need the following definitions.

For a set $B$, we denote by $\bar{B}$ the closure of $B$. We say that a fuzzy set $A$, from $\mathbb{R}^{+}=(0, \infty)$ into the interval $[0,1]$, is a fuzzy number, if $A$ is normal, convex, upper semicontinuous (see [14]), and the support $\operatorname{supp} A=\overline{\bigcup_{a \in(0,1]}[A]_{a}}=\overline{\{x: A(x)>0\}}$ is compact. Then from $\left[12\right.$, Theorems 3.1.5 and 3.1.8], the $a$-cuts of the fuzzy number $A,[A]_{a}=\left\{x \in \mathbb{R}^{+}\right.$: $A(x) \geq a\}$, are closed intervals.

We say that a fuzzy number $A$ is positive if $\operatorname{supp} A \subset(0, \infty)$.

It is obvious that if $A$ is a positive real number, then $A$ is a positive fuzzy number and $[A]_{a}=[A, A], a \in(0,1]$. In this case, we say that $A$ is a trivial fuzzy number.

We say that $x_{n}$ is a positive solution of (1.1) if $x_{n}$ is a sequence of positive fuzzy numbers which satisfies (1.1).

A positive fuzzy number $x$ is a positive equilibrium for (1.1) if

$$
x=A+\frac{\sum_{i=1}^{k} c_{i} x}{\sum_{j=1}^{m} d_{j} x} .
$$

Let $E, H$ be fuzzy numbers with

$$
[E]_{a}=\left[E_{l, a}, E_{r, a}\right], \quad[H]_{a}=\left[H_{l, a}, H_{r, a}\right], \quad a \in(0,1] .
$$

According to [10] and [13, Lemma 2.3], we have that $\operatorname{MIN}\{E, H\}=E$ if

$$
E_{l, a} \leq H_{l, a}, \quad E_{r, a} \leq H_{r, a}, \quad a \in(0,1] .
$$

Moreover, let $c_{i}, f_{i}, d_{j}, g_{j}, i=1,2, \ldots, k, j=1,2, \ldots, m$, be positive fuzzy numbers such that for $a \in(0,1]$,

$$
\begin{array}{rr}
{\left[c_{i}\right]_{a}=\left[c_{i, l, a}, c_{i, r, a}\right],} & {\left[f_{i}\right]_{a}=\left[f_{i, l, a}, f_{i, r, a}\right],} \\
{\left[d_{j}\right]_{a}=\left[d_{j, l, a}, d_{j, r, a}\right],} & {\left[g_{j}\right]_{a}=\left[g_{j, l, a}, g_{j, r, a}\right],} \\
E=\frac{\sum_{i=1}^{k} c_{i}}{\sum_{j=1}^{m} d_{j}}, & H=\frac{\sum_{i=1}^{k} f_{i}}{\sum_{j=1}^{m} g_{j}} .
\end{array}
$$

We will say that $E$ is less than $H$ and we will write

$$
E \prec H
$$

if

$$
\frac{\sum_{i=1}^{k} \sup _{a \in(0,1]} c_{i, r, a}}{\sum_{j=1}^{m} \inf _{a \in(0,1]} d_{j, l, a}}<\frac{\sum_{i=1}^{k} \inf _{a \in(0,1]} f_{i, l, a}}{\sum_{j=1}^{m} \sup _{a \in(0,1]} g_{j, r, a}}
$$


In addition, we will say that $E$ is equal to $H$ and we will write

$$
E \doteq H \quad \text { if } E \prec H, H \prec E,
$$

which means that for $i=1,2, \ldots, k, j=1,2, \ldots, m$, and $a \in(0,1]$,

$$
c_{i, l, a}=c_{i, r, a}, \quad f_{i, l, a}=f_{i, r, a}, \quad d_{j, l, a}=d_{j, r, a}, \quad g_{j, l, a}=g_{j, r, a},
$$

and so

$$
E_{l, a}=E_{r, a}=H_{l, a}=H_{r, a}, \quad a \in(0,1],
$$

which imples that $E, H$ are equal real numbers.

For the fuzzy numbers $E, H$, we give the metric (see $[9,17,18]$ )

$$
D(E, H)=\sup \max \left\{\left|E_{l, a}-H_{l, a}\right|,\left|E_{r, a}-H_{r, a}\right|\right\},
$$

where sup is taken for all $a \in(0,1]$.

The fuzzy analog of boundedness and persistence $($ see $[5,6])$ is given as follows: we say that a sequence of positive fuzzy numbers $x_{n}$ persists (resp., is bounded) if there exists a positive number $M$ (resp., $N$ ) such that

$$
\operatorname{supp} x_{n} \subset[M, \infty) \quad\left(\text { resp., } \operatorname{supp} x_{n} \subset(0, N]\right), \quad n=1,2, \ldots
$$

In addition, we say that $x_{n}$ is bounded and persists if there exist numbers $M, N \in(0, \infty)$ such that

$$
\operatorname{supp} x_{n} \subset[M, N], \quad n=1,2, \ldots
$$

Let $x_{n}$ be a sequence of positive fuzzy numbers such that

$$
\left[x_{n}\right]_{a}=\left[L_{n, a}, R_{n, a}\right], \quad a \in(0,1], n=0,1, \ldots,
$$

and let $x$ be a positive fuzzy number such that

$$
[x]_{a}=\left[L_{a}, R_{a}\right], \quad a \in(0,1] .
$$

We say that $x_{n}$ nearly converges to $x$ with respect to $D$ as $n \rightarrow \infty$ if for every $\delta>0$, there exists a measurable set $T, T \subset(0,1]$, of measure less than $\delta$ such that

$$
\lim D_{T}\left(x_{n}, x\right)=0, \quad \text { as } n \longrightarrow \infty,
$$

where

$$
D_{T}\left(x_{n}, x\right)=\sup _{a \in(0,1]-T}\left\{\max \left\{\left|L_{n, a}-L_{a}\right|,\left|R_{n, a}-R_{a}\right|\right\}\right\} .
$$

If $T=\varnothing$, we say that $x_{n}$ converges to $x$ with respect to $D$ as $n \rightarrow \infty$. 
Let $X$ be the set of positive fuzzy numbers. Let $E, H \in X$. From [18, Theorem 2.1], we have that $E_{l, a}, H_{l, a}$ (resp., $E_{r, a}, H_{r, a}$ ) are increasing (resp., decreasing) functions on $(0,1]$. Therefore, using the definition of the fuzzy numbers, there exist the Lebesque integrals

$$
\int_{J}\left|E_{l, a}-H_{l, a}\right| d a, \quad \int_{J}\left|E_{r, a}-H_{r, a}\right| d a,
$$

where $J=(0,1]$. We define the function $D_{1}: X \times X \rightarrow R^{+}$such that

$$
D_{1}(E, H)=\max \left\{\int_{J}\left|E_{l, a}-H_{l, a}\right| d a, \int_{J}\left|E_{r, a}-H_{r, a}\right| d a\right\} .
$$

If $D_{1}(E, H)=0$, we have that there exists a measurable set $T$ of measure zero such that

$$
E_{l, a}=H_{l, a}, \quad E_{r, a}=H_{r, a} \quad \forall a \in(0,1]-T
$$

We consider, however, two fuzzy numbers $E, H$ to be equivalent if there exists a measurable set $T$ of measure zero such that (2.20) hold and if we do not distinguish between equivalence of fuzzy numbers, then $X$ becomes a metric space with metric $D_{1}$.

We say that a sequence of positive fuzzy numbers $x_{n}$ converges to a positive fuzzy number $x$ with respect to $D_{1}$ as $n \rightarrow \infty$ if

$$
\lim D_{1}\left(x_{n}, x\right)=0, \quad \text { as } n \longrightarrow \infty .
$$

We define the fuzzy analog for periodicity (see [11]) as follows.

A sequence $\left\{x_{n}\right\}$ of positive fuzzy numbers $x_{n}$ is said to be periodic of period $p$ if

$$
D\left(x_{n+p}, x_{n}\right)=0, \quad n=0,1, \ldots
$$

Suppose that (1.1) has a unique positive equilibrium $x$. We say that the positive equilibrium $x$ of (1.1) is stable if for every $\epsilon>0$, there exists a $\delta=\delta(\epsilon)$ such that for every positive solution $x_{n}$ of $(1.1)$ which satisfies $D\left(x_{-i}, x\right) \leq \delta, i=0,1, \ldots, \pi$, we have $D\left(x_{n}, x\right) \leq \epsilon$ for all $n \geq 0$.

Moreover, we say that the positive equilibrium $x$ of (1.1) is nearly asymptotically stable if it is stable and every positive solution of (1.1) nearly tends to the positive equilibrium of (1.1) with respect to $D$ as $n \rightarrow \infty$.

Finally, we give the fuzzy analog of the concept of oscillation (see [11]). Let $x_{n}$ be a sequence of positive fuzzy numbers and let $x$ be a positive fuzzy number. We say that $x_{n}$ oscillates about $x$ if for every $n_{0} \in \mathbb{N}$, there exist $s, m \in \mathbb{N}, s, m \geq n_{0}$, such that

$$
\operatorname{MIN}\left\{x_{m}, x\right\}=x_{m}, \quad \operatorname{MIN}\left\{x_{s}, x\right\}=x
$$

or

$$
\operatorname{MIN}\left\{x_{m}, x\right\}=x, \quad \operatorname{MIN}\left\{x_{s}, x\right\}=x_{s}
$$




\section{Main results}

Arguing as in $[13,14,15]$, we can easily prove the following proposition which concerns the existence and the uniqueness of the positive solutions of (1.1).

Proposition 3.1. Consider (1.1), where $k, m \in\{1,2, \ldots\}, A, c_{i}, d_{j}, i \in\{1,2, \ldots, k\}, j \in\{1$, $2, \ldots, m\}$, are positive fuzzy numbers, and $p_{i}, q_{j}, i \in\{1,2, \ldots, k\}, j \in\{1,2, \ldots, m\}$, are positive integers. Then for any positive fuzzy numbers $x_{-\pi}, x_{-\pi+1}, \ldots, x_{0}$, there exists a unique positive solution $x_{n}$ of (1.1) with initial values $x_{-\pi}, x_{-\pi+1}, \ldots, x_{0}$.

Now, we present conditions so that (1.1) has unbounded solutions.

Proposition 3.2. Consider (1.1), where $k, m \in\{1,2, \ldots\}, A, c_{i}, d_{j}, i \in\{1,2, \ldots, k\}, j \in\{1$, $2, \ldots, m\}$, are positive fuzzy numbers, and $p_{i}, i \in\{1,2, \ldots, k\}, q_{j}, j \in\{1,2, \ldots, m\}$, are positive integers. If

$$
A \prec G, \quad G=\frac{\sum_{i=1}^{k} c_{i}}{\sum_{j=1}^{m} d_{j}},
$$

then (1.1) has unbounded solutions.

Proof. Let

$$
[A]_{a}=\left[A_{l, a}, A_{r, a}\right], \quad a \in(0,1]
$$

From (2.4) and (3.2) and since $A, c_{i}, d_{j}, i=1,2, \ldots, k, j=1,2, \ldots, m$, are positive fuzzy numbers, there exist positive real numbers $B, C, a_{i}, e_{i}, h_{j}, b_{j}, i=1,2, \ldots, k, j=1,2, \ldots, m$, such that

$$
\begin{array}{lll}
B=\inf _{a \in(0,1]} A_{l, a}, & C=\sup _{a \in(0,1]} A_{r, a}, & a_{i}=\inf _{a \in(0,1]} c_{i, l, a}, \\
e_{i}=\sup _{a \in(0,1]} c_{i, r, a}, & h_{j}=\inf _{a \in(0,1]} d_{j, l, a}, & b_{j}=\sup _{a \in(0,1]} d_{j, r, a} .
\end{array}
$$

Let $x_{n}$ be a positive solution of (1.1) such that (2.14) hold and the initial values $x_{i}, i=$ $-\pi,-\pi+1, \ldots, 0$, are positive fuzzy numbers which satisfy

$$
\left[x_{i}\right]_{a}=\left[L_{i, a}, R_{i, a}\right], \quad i=-\pi,-\pi+1, \ldots, 0, a \in(0,1]
$$

and for a fixed $\bar{a} \in(0,1]$, the relations

$$
R_{i, \bar{a}}>\frac{Z^{2}}{W-C}, \quad L_{i, \bar{a}}<W, \quad i=-\pi,-\pi+1, \ldots, 0,
$$

are satisfied, where

$$
Z=\frac{\sum_{i=1}^{k} e_{i}}{\sum_{j=1}^{m} h_{j}}, \quad W=\frac{\sum_{i=1}^{k} a_{i}}{\sum_{j=1}^{m} b_{j}}
$$


Using [15, Lemma 1], we can easily prove that $L_{n, a}, R_{n, a}$ satisfy the family of systems of parametric ordinary difference equations

$$
\begin{aligned}
L_{n+1, a} & =A_{l, a}+\frac{\sum_{i=1}^{k} c_{i, l, a} L_{n-p_{i}, a}}{\sum_{j=1}^{m} d_{j, r, a} R_{n-q_{j}, a}}, \quad n=0,1, \ldots \\
R_{n+1, a} & =A_{r, a}+\frac{\sum_{i=1}^{k} c_{i, r, a} R_{n-p_{i}, a}}{\sum_{j=1}^{m} d_{j, l, a} L_{n-q_{j}, a}},
\end{aligned}
$$

Since (3.1) holds, it is obvious that

$$
A_{l, \bar{a}}<\frac{\sum_{i=1}^{k} c_{i, r, \bar{a}}}{\sum_{j=1}^{m} d_{j, l, \bar{a}}} .
$$

Using (3.8) and applying [16, Proposition 1] to the system (3.7) for $a=\bar{a}$, we have that

$$
\lim _{n \rightarrow \infty} L_{n, \bar{a}=A_{l, \bar{a}}}, \quad \lim _{n \rightarrow \infty} R_{n, \bar{a}}=\infty .
$$

Therefore, from (3.9), the solution $x_{n}$ of (1.1) which satisfies (3.4) and (3.5) is unbounded.

Remark 3.3. From the proof of Proposition 3.2, it is obvious that (1.1) has unbounded solutions if there exists at least one $a \in(0,1]$ such that $(3.8)$ holds.

In the following proposition, we study the boundedness and persistence of the positive solutions of (1.1).

Proposition 3.4. Consider (1.1), where $k, m \in\{1,2, \ldots\}, A, c_{i}, d_{j}, i \in\{1,2, \ldots, k\}, j \in$ $\{1,2, \ldots, m\}$, are positive fuzzy numbers, and $p_{i}, i \in\{1,2, \ldots, k\}, q_{j}, j \in\{1,2, \ldots, m\}$, are positive integers. If either

$$
A \doteq G
$$

or

$$
G \prec A
$$

holds, then every positive solution of (1.1) is bounded and persists.

Proof. Firstly, suppose that (3.10) is satisfied; then $A, c_{i}, d_{j}, i=1,2, \ldots, k, j=1,2, \ldots, m$, are positive real numbers. Hence, for $i=1,2, \ldots, k, j=1,2, \ldots, m$, we get

$$
\begin{gathered}
A=A_{l, a}=A_{r, a}, \quad c_{i}=c_{i, l, a}=c_{i, r, a}, \quad d_{j}=d_{j, l, a}=d_{j, r, a}, \quad a \in(0,1], \\
A=\frac{\sum_{i=1}^{k} c_{i}}{\sum_{j=1}^{m} d_{j}} .
\end{gathered}
$$


Let $x_{n}$ be a positive solution of (1.1) such that (2.14) hold and let $x_{i}, i=-\pi,-\pi+$ $1, \ldots, 0$, be the positive initial values of $x_{n}$ such that (3.4) hold. Then there exist positive numbers $T_{i}, S_{i}, i=-\pi,-\pi+1, \ldots, 0$, such that

$$
T_{i} \leq L_{i, a}, R_{i, a} \leq S_{i}, \quad i=-\pi,-\pi+1, \ldots, 0
$$

Let $\left(y_{n}, z_{n}\right)$ be the positive solution of the system of ordinary difference equations

$$
y_{n+1}=A+\frac{\sum_{i=1}^{k} c_{i} y_{n-p_{i}}}{\sum_{j=1}^{m} d_{j} z_{n-q_{j}}}, \quad z_{n+1}=A+\frac{\sum_{i=1}^{k} c_{i} z_{n-p_{i}}}{\sum_{j=1}^{m} d_{j} y_{n-q_{j}}},
$$

with initial values $\left(y_{i}, z_{i}\right), i=-\pi,-\pi+1, \ldots, 0$, such that $y_{i}=T_{i}, z_{i}=S_{i}, i=-\pi,-\pi+$ $1, \ldots, 0$. Then from (3.14) and (3.15), we can easily prove that

$$
y_{1} \leq L_{1, a}, \quad R_{1, a} \leq z_{1}, \quad a \in(0,1]
$$

and working inductively, we take

$$
y_{n} \leq L_{n, a}, \quad R_{n, a} \leq z_{n}, \quad n=1,2, \ldots, a \in(0,1]
$$

Since from (3.13) and [16, Proposition 3], $\left(y_{n}, z_{n}\right)$ is bounded and persists, from (3.17), it is obvious that $x_{n}$ is also bounded and persists.

Now, suppose that (3.11) holds; then

$$
B>Z, \quad C>W .
$$

We concider the system of ordinary difference equations

$$
y_{n+1}=B+\frac{\sum_{i=1}^{k} a_{i} y_{n-p_{i}}}{\sum_{j=1}^{m} b_{j} z_{n-q_{j}}}, \quad z_{n+1}=C+\frac{\sum_{i=1}^{k} e_{i} z_{n-p_{i}}}{\sum_{j=1}^{m} h_{j} y_{n-q_{j}}},
$$

where $B, C, a_{i}, e_{i}, b_{j}, h_{j}, i=1,2, \ldots, k, j=1,2, \ldots, m$, are defined in (3.3).

Let $\left(y_{n}, z_{n}\right)$ be a solution of (3.19) with initial values $\left(y_{i}, z_{i}\right), i=-\pi,-\pi+1, \ldots, 0$, such that $y_{i}=T_{i}, z_{i}=S_{i}, i=-\pi,-\pi+1, \ldots, 0$, where $T_{i}, S_{i}, i=-\pi,-\pi+1, \ldots, 0$, are defined in (3.14). Arguing as above, we can prove that (3.17) holds. Since from (3.18) and [16, Proposition 3], $\left(y_{n}, z_{n}\right)$ is bounded and persists, then from (3.17), it is obvious that, $x_{n}$ is also bounded and persists. This completes the proof of the proposition.

In what follows, we need the following lemmas.

Lemma 3.5. Let $r_{i}, s_{j}, i=1,2, \ldots, k, j=1,2, \ldots, m$, be positive integers such that

$$
\left(r_{1}, r_{2}, \ldots, r_{k}, s_{1}, s_{2}, \ldots, s_{m}\right)=1
$$

where $\left(r_{1}, r_{2}, \ldots, r_{k}, s_{1}, s_{2}, \ldots, s_{m}\right)$ is the greatest common divisor of the integers $r_{i}, s_{j}, i=1,2$, $\ldots, k, j=1,2, \ldots, m$. Then the following statements are true. 
(I) There exists an even positive integer $w_{1}$ such that for any nonnegative integer $p$, there exist nonnegative integers $\alpha_{i p}, \beta_{j p}, i=1,2, \ldots, k, j=1,2, \ldots, m$, such that

$$
\sum_{i=1}^{k} \alpha_{i p} r_{i}+\sum_{j=1}^{m} \beta_{j p} s_{j}=w_{1}+2 p, \quad p=0,1, \ldots
$$

where $\sum_{j=1}^{m} \beta_{j p}$ is an even integer.

(II) Suppose that all $r_{i}, i=1,2, \ldots, k$, are not even and all $s_{j}, j=1,2, \ldots, m$, are not odd integers. Then there exists an odd positive integer $w_{2}$ such that for any nonnegative integer $p$, there exist nonnegative integers $\gamma_{i p}, \delta_{j p}, i=1,2, \ldots, k, j=1,2, \ldots, m$, such that

$$
\sum_{i=1}^{k} \gamma_{i p} r_{i}+\sum_{j=1}^{m} \delta_{j p} s_{j}=w_{2}+2 p, \quad p=0,1, \ldots
$$

where $\sum_{j=1}^{m} \delta_{j p}$ is an even integer.

(III) Suppose that all $r_{i}, i=1,2, \ldots, k$, are not even and all $s_{j}, j=1,2, \ldots, m$, are not odd integers. Then there exists an even positive integer $w_{3}$ such that for any nonnegative integer $p$, there exist nonnegative integers $\epsilon_{i p}, \xi_{j p}, i=1,2, \ldots, k, j=1,2, \ldots, m$, such that

$$
\sum_{i=1}^{k} \epsilon_{i p} r_{i}+\sum_{j=1}^{m} \xi_{j p} s_{j}=w_{3}+2 p, \quad p=0,1, \ldots,
$$

where $\sum_{j=1}^{m} \xi_{j p}$ is an odd integer.

(IV) There exists an odd positive integer $w_{4}$ such that for any nonnegative integer $p$, there exist nonnegative integers $\lambda_{i p}, \mu_{j p}, i=1,2, \ldots, k, j=1,2, \ldots, m$, such that

$$
\sum_{i=1}^{k} \lambda_{i p} r_{i}+\sum_{j=1}^{m} \mu_{j p} s_{j}=w_{4}+2 p, \quad p=0,1, \ldots
$$

where $\sum_{j=1}^{m} \mu_{j p}$ is an odd integer.

Proof. (I) Since(3.20) holds, there exist integers $\eta_{i}, \iota_{j}, i=1,2, \ldots, k, j=1,2, \ldots, m$, such that

$$
\sum_{i=1}^{k} \eta_{i} r_{i}+\sum_{j=1}^{m} \iota_{j} s_{j}=1
$$

If for any real number $a$, we denote by $[a]$ the integral part of $a$, we set for $i=2,3, \ldots, k$, $j=1,2, \ldots, m$,

$$
\begin{aligned}
& \alpha_{1 p}=2 p \eta_{1}+2 \sum_{i=2}^{k} r_{i}+2 \sum_{j=1}^{m} s_{j}-2 \sum_{i=2}^{k} g_{i p} r_{i}-2 \sum_{j=1}^{m} h_{j p} s_{j}, \\
& \alpha_{i p}=2 p \eta_{i}+2 g_{i p} r_{1}, \quad \beta_{j p}=2 p \iota_{j}+2 h_{j p} r_{1},
\end{aligned}
$$


where

$$
g_{i p}=\left[\frac{-p \eta_{i}}{r_{1}}\right]+1, \quad h_{j p}=\left[\frac{-p \iota_{j}}{r_{1}}\right]+1, \quad i=2,3, \ldots, k, j=1,2, \ldots, m .
$$

Therefore, from (3.25) and (3.26), we can easily prove that $\alpha_{i p}, \beta_{j p}, i=1,2, \ldots, k, j=$ $1,2, \ldots, m$, which are defined in (3.26), are positive integers satisfying (3.21) for

$$
w_{1}=2 r_{1}\left(\sum_{i=2}^{k} r_{i}+\sum_{j=1}^{m} s_{j}\right)
$$

and $\sum_{j=1}^{m} \beta_{j p}$ is an even number.

(II) Firstly, suppose that one of $r_{i}, i=1,2, \ldots, k$, is an odd positive integer and without loss of generality, let $r_{1}$ be an odd positive integer. Relation (3.22) follows immediately if we set for $i=2, \ldots, k$ and for $j=1,2, \ldots, m$,

$$
\gamma_{1 p}=\alpha_{1 p}+1, \quad \gamma_{i p}=\alpha_{i p}, \quad \delta_{j p}=\beta_{j p}, \quad w_{2}=w_{1}+r_{1} .
$$

Now, suppose that $r_{i}, i=1,2, \ldots, k$, are even positive integers; then from (3.20), one of $s_{j}, j=1,2, \ldots, m$, is an odd positive integer and from the hypothesis, one of $s_{j}, j=$ $1,2, \ldots, m$, is an even positive integer. Without loss of generality, let $s_{1}$ be an odd positive integer and $s_{2}$ be an even positive integer. Relation (3.22) follows immediately if we set for $i=1,2, \ldots, k$ and for $j=3, \ldots, m$,

$$
\gamma_{i p}=\alpha_{i p}, \quad \delta_{1 p}=\beta_{1 p}+1, \quad \delta_{2 p}=\beta_{2 p}+1, \quad \delta_{j p}=\beta_{j p}, \quad w_{2}=w_{1}+s_{1}+s_{2} .
$$

(III) Firstly, suppose that one of $s_{j}, j=1,2, \ldots, m$, is an even positive integer and without loss of generality, let $s_{1}$ be an even positive integer. Relation (3.23) follows immediately if we set for $i=1,2, \ldots, k$ and $j=2, \ldots, m$,

$$
\epsilon_{i p}=\alpha_{i p}, \quad \xi_{1 p}=\beta_{1 p}+1, \quad \xi_{j p}=\beta_{j p}, \quad w_{3}=w_{1}+s_{1} .
$$

Now, suppose that $s_{j}, j=1,2, \ldots, m$, are odd positive integers; then from the hypothesis, at least one of $r_{i}, i=1,2, \ldots, k$, is an odd positive integer, and without loss of generality, let $r_{1}$ be an odd integer. Relation (3.23) follows immediately if we set for $i=2, \ldots, k$, $j=2,3, \ldots, m$,

$$
\epsilon_{1 p}=\alpha_{1 p}+1, \quad \epsilon_{i p}=\alpha_{i p}, \quad \delta_{1 p}=\beta_{1 p}+1, \quad \delta_{j p}=\beta_{j p}, \quad w_{3}=w_{1}+s_{1}+r_{1} .
$$

(IV) Firstly, suppose that at least one of $s_{j}, j=1,2, \ldots, m$, is an odd positive integer and without loss of generality, let $s_{1}$ be an odd positive integer. Relation (3.24) follows immediately if we set for $i=1,2, \ldots, k, j=2,3, \ldots, m$,

$$
\lambda_{i p}=\alpha_{i p}, \quad \mu_{1 p}=\beta_{1 p}+1, \quad \mu_{j p}=\beta_{j p}, \quad w_{4}=w_{1}+s_{1} .
$$


Now, suppose that $s_{j}, j=1,2, \ldots, m$, are even positive integers; then from (3.20), at least one of $r_{i}, i=1,2, \ldots, k$, is an odd positive integer, and without loss of generality, let $r_{1}$ be an odd positive integer. Relation (3.24) follows immediately if we set for $i=2,3, \ldots, k$, $j=2,3, \ldots, m$,

$\lambda_{1 p}=\alpha_{1 p}+1, \quad \lambda_{i p}=\alpha_{i p}, \quad \mu_{1 p}=\beta_{1 p}+r_{1}, \quad \mu_{j p}=\beta_{j p}, \quad w_{4}=w_{1}+r_{1}\left(s_{1}+1\right)$.

This completes the proof of the lemma.

Lemma 3.6. Consider system (3.19), where B, C are positive constants such that

$$
B=\frac{\sum_{i=1}^{k} e_{i}}{\sum_{j=1}^{m} h_{j}}, \quad C=\frac{\sum_{i=1}^{k} a_{i}}{\sum_{j=1}^{m} b_{j}}
$$

Then the following statements are true.

(I) Let $r$ be a common divisor of the integers $p_{i}+1, q_{j}+1, i=1,2, \ldots, k, j=1,2, \ldots, m$, such that

$$
p_{i}+1=r r_{i}, \quad i=1,2, \ldots, k, \quad q_{j}+1=r s_{j}, \quad j=1,2, \ldots, m
$$

then system (3.19) has periodic solutions of prime period $r$. Moreover, if all $r_{i}, i=1,2, \ldots, k$, (resp., $s_{j}, j=1,2, \ldots, m$ ) are even (resp., odd) positive integers, then system (3.19) has periodic solutions of prime period $2 r$.

(II) Let $r$ be the greatest common divisor of the integers $p_{i}+1, q_{j}+1, i=1,2, \ldots, k, j=$ $1,2, \ldots, m$, such that (3.36) hold; then if all $r_{i}, i=1,2, \ldots, k$, (resp., $\left.s_{j}, j=1,2, \ldots, m\right)$ are even (resp., odd) positive integers, every positive solution of (3.19) tends to a periodic solution of period 2r; otherwise, every positive solution of (3.19) tends to a periodic solution of period $r$.

Proof. (I) From relations (3.35), (3.36), and [16, Proposition 2], system (3.19) has periodic solutions of prime period $r$.

Now, we prove that system (3.19) has periodic solutions of prime period $2 r$, if all $r_{i}$, $i=1,2, \ldots, k$, (resp., $s_{j}, j=1,2, \ldots, m$ ) are even (resp., odd) positive integers.

Suppose first that $p_{k}<q_{m}$. Let $\left(y_{n}, z_{n}\right)$ be a positive solution of (3.19) with initial values satisfying

$$
\begin{gathered}
y_{-r s_{m}+2 r \lambda+\zeta}=y_{-r+\zeta}, \quad z_{-r s_{m}+2 r \lambda+\zeta}=z_{-r+\zeta}, \\
y_{-r s_{m}+2 r v+r+\zeta}=y_{-2 r+\zeta}, \quad z_{-r s_{m}+2 r \nu+r+\zeta}=z_{-2 r+\zeta}, \\
\lambda=0,1, \ldots, \frac{s_{m}-1}{2}, \quad v=0,1, \ldots, \frac{s_{m}-3}{2}, \quad \zeta=1,2, \ldots, r
\end{gathered}
$$

and, in addition, for $\zeta=1,2, \ldots, r$,

$$
y_{-2 r+\zeta}>B, \quad y_{-r+\zeta}>B, \quad z_{-r+\zeta}=\frac{C y_{-2 r+\zeta}}{y_{-2 r+\zeta}-B}, \quad z_{-2 r+\zeta}=\frac{C y_{-r+\zeta}}{y_{-r+\zeta}-B}
$$


From (3.19), (3.35), (3.36), (3.37), and (3.38), we get for $\zeta=1,2, \ldots, r$,

$$
\begin{array}{ll}
y_{\zeta}=B+C \frac{y_{-2 r+\zeta}}{z_{-r+\zeta}}=y_{-2 r+\zeta}, & z_{\zeta}=C+B \frac{z_{-2 r+\zeta}}{y_{-r+\zeta}}=z_{-2 r+\zeta}, \\
y_{r+\zeta}=B+C \frac{y_{-r+\zeta}}{z_{-2 r+\zeta}}=y_{-r+\zeta}, & z_{r+\zeta}=C+B \frac{z_{-r+\zeta}}{y_{-2 r+\zeta}}=z_{-r+\zeta} .
\end{array}
$$

Let a $v \in\{2,3, \ldots\}$. Suppose that for all $u=1,2, \ldots, v-1$ and $\zeta=1,2, \ldots, r$, we have

$$
y_{2 u r+\zeta}=y_{-2 r+\zeta}, \quad z_{2 u r+\zeta}=z_{-2 r+\zeta}, \quad y_{2 u r+r+\zeta}=y_{-r+\zeta}, \quad z_{2 u r+r+\zeta}=z_{-r+\zeta} .
$$

Then from (3.19), (3.35)-(3.40), we get for $\zeta=1,2, \ldots, r$,

$$
y_{2 v r+\zeta}=B+C \frac{y_{-2 r+\zeta}}{z_{-r+\zeta}}=y_{-2 r+\zeta}
$$

Similarly, we can prove that for $\zeta=1,2, \ldots, r$,

$$
z_{2 v r+\zeta}=z_{-2 r+\zeta}, \quad y_{2 v r+r+\zeta}=y_{-r+\zeta}, \quad z_{2 v r+r+\zeta}=z_{-r+\zeta}
$$

Therefore, from (3.39)-(3.42), we have that system (3.19) has periodic solutions of period $2 r$.

Now, suppose that $q_{m}<p_{k}$. Let $\left(y_{n}, z_{n}\right)$ be a positive solution of (3.19) such that the initial values satisfy relations (3.38) and for $\omega=0,1, \ldots, r_{k} / 2-1, \theta=1,2, \ldots, 2 r$,

$$
y_{-r r_{k}+2 r \omega+\theta}=y_{-2 r+\theta}, \quad z_{-r r_{k}+2 r \omega+\theta}=z_{-2 r+\theta} .
$$

Then arguing as above, we can easily prove that $\left(y_{n}, z_{n}\right)$ is a periodic solution of period $2 r$. This completes the proof of statement (I).

(II) Now, we prove that every positive solution of system (3.19) tends to a periodic solution of period $\kappa r$, where

$$
\kappa= \begin{cases}2 & \text { if } r_{i}, i=1,2, \ldots, k, \text { are even }, s_{j}, j=1,2, \ldots, m, \text { are odd } \\ 1 & \text { otherwise }\end{cases}
$$

Let $\left(y_{n}, z_{n}\right)$ be an arbitrary positive solution of (3.19). We prove that there exist the

$$
\lim _{n \rightarrow \infty} y_{\kappa n r+i}=\epsilon_{i}, \quad i=0,1, \ldots, \kappa r-1 .
$$

We fix a $\tau \in\{0,1, \ldots, \kappa r-1\}$. Since from [16, Proposition 3], the solution $\left(y_{n}, z_{n}\right)$ is bounded and persists, we have

$$
\begin{array}{ll}
\liminf _{n \rightarrow \infty} y_{\kappa n r+\tau}=l_{\tau} \geq B, & \liminf _{n \rightarrow \infty} z_{\kappa n r+\tau}=m_{\tau} \geq C, \\
\limsup _{n \rightarrow \infty} y_{\kappa n r+\tau}=L_{\tau}<\infty, & \limsup _{n \rightarrow \infty} z_{\kappa n r+\tau}=M_{\tau}<\infty .
\end{array}
$$

Therefore, from relations (3.19), (3.35), and (3.46), we take

$$
m_{\tau}=\frac{C L_{\tau}}{L_{\tau}-B}, \quad l_{\tau}=\frac{B M_{\tau}}{M_{\tau}-C} .
$$


We prove that (3.45) is true for $i=\tau$. Suppose on the contrary that $l_{\tau}<L_{\tau}$. Then from (3.46), there exists an $\epsilon>0$ such that

$$
L_{\tau}>l_{\tau}+\epsilon>B+\epsilon
$$

In view of (3.46), there exists a sequence $n_{\mu}, \mu=1,2, \ldots$, such that

$$
\begin{gathered}
\lim _{\mu \rightarrow \infty} y_{\kappa r n_{\mu}+\tau}=L_{\tau}, \quad \lim _{\mu \rightarrow \infty} y_{r\left(\kappa n_{\mu}-r_{i}\right)+\tau}=T_{r_{i}, \tau} \leq L_{\tau}, \\
\lim _{\mu \rightarrow \infty} z_{r\left(\kappa n_{\mu}-s_{j}\right)+\tau}=S_{s_{j}, \tau} \geq m_{\tau} .
\end{gathered}
$$

In view of (3.19), (3.35), (3.46), (3.47), and (3.49), we take

$$
L_{\tau}=B+\frac{\sum_{i=1}^{k} a_{i} T_{r_{i}, \tau}}{\sum_{j=1}^{m} b_{j} S_{s_{j}, \tau}} \leq B+\frac{C L_{\tau}}{m_{\tau}}=L_{\tau}
$$

and obviously, we have that

$$
\begin{aligned}
& T_{r_{i}, \tau}=L_{\tau}, \quad i=1,2, \ldots, k \\
& S_{s_{j}, \tau}=m_{\tau}, \quad j=1,2, \ldots, m .
\end{aligned}
$$

In addition, using (3.19), (3.35), (3.46), (3.47), and (3.51), for $\kappa=2$, from statements (I) and (IV) of Lemma 3.5 and arguing as above, we take for $\gamma=0,1, \ldots$,

$$
\lim _{\mu \rightarrow \infty} y_{r\left(2 n_{\mu}-w_{1}-2 \gamma\right)+\tau}=L_{\tau}, \quad \lim _{\mu \rightarrow \infty} z_{r\left(2 n_{\mu}-w_{1}-s_{1}-2 \gamma\right)+\tau}=m_{\tau}
$$

and for $\kappa=1$ and from all the statements of Lemma 3.5,

$$
\begin{array}{ll}
\lim _{\mu \rightarrow \infty} y_{r\left(n_{\mu}-w_{1}-2 \gamma\right)+\tau}=L_{\tau}, & \lim _{\mu \rightarrow \infty} y_{r\left(n_{\mu}-w_{2}-2 \gamma\right)+\tau}=L_{\tau}, \\
\lim _{\mu \rightarrow \infty} z_{r\left(n_{\mu}-w_{3}-2 \gamma\right)+\tau}=m_{\tau}, & \lim _{\mu \rightarrow \infty} z_{r\left(n_{\mu}-w_{4}-2 \gamma\right)+\tau}=m_{\tau},
\end{array}
$$

$w_{1}, w_{2}, w_{3}, w_{4}$ are defined in Lemma 3.5.

Let a $\sigma_{\kappa} \in\{0,1, \ldots,(3-\kappa) \phi\}, \phi=\max \left\{r_{k}, s_{m}\right\}$. Suppose first that $\kappa=2$. Then in view of (3.19), there exist positive integers $p, q$ and a continuous function $F_{\sigma_{2}}: \mathbb{R} \times \mathbb{R} \times \cdots \times$ $\mathbb{R} \rightarrow \mathbb{R}$ such that

$$
y_{r\left(2 n_{\mu}+2 \sigma_{2}\right)+\tau}=B+F_{\sigma_{2}}\left(\zeta_{n_{\mu}, 0}, \ldots, \zeta_{n_{\mu}, p}, \xi_{n_{\mu}, 0}, \ldots, \xi_{n_{\mu}, q}\right),
$$

where for $i=0,1, \ldots, p, j=0,1, \ldots, q$,

$$
\zeta_{n_{\mu}, i}=y_{r\left(2 n_{\mu}-w_{1}-2 i\right)+\tau}, \quad \xi_{n_{\mu}, j}=z_{r\left(2 n_{\mu}-w_{1}-s_{1}-2 j\right)+\tau} .
$$

If $\kappa=1$, there exist positive integers $v_{1}, v_{2}, v_{3}, v_{4}$ and a continuous function $G_{\sigma_{1}}: \mathbb{R} \times$ $\mathbb{R} \times \cdots \times \mathbb{R} \rightarrow \mathbb{R}$ such that

$$
y_{r\left(n_{\mu}+\sigma_{1}\right)+\tau}=B+G_{\sigma_{1}}\left(\zeta_{n_{\mu}, 0}, \ldots, \zeta_{n_{\mu}, v_{1}}, \bar{\zeta}_{n_{\mu}, 0}, \ldots, \bar{\zeta}_{n_{\mu}, v_{2}}, \xi_{n_{\mu}, 0}, \ldots, \xi_{n_{\mu}, v_{3}}, \bar{\xi}_{n_{\mu}, 0}, \ldots, \bar{\xi}_{n_{\mu}, v_{4}}\right),
$$


where for $i=0,1, \ldots, v_{1}, \bar{i}=0,1, \ldots, v_{2}, j=0,1, \ldots, v_{3}$, and $\bar{j}=0,1, \ldots, v_{4}$,

$$
\begin{array}{ll}
\zeta_{n_{\mu}, i}=y_{r\left(n_{\mu}-w_{1}-2 i\right)+\tau}, & \bar{\zeta}_{n_{\mu}, \bar{i}}=y_{r\left(n_{\mu}-w_{2}-2 \bar{i}\right)+\tau}, \\
\xi_{n_{\mu}, j}=z_{r\left(n_{\mu}-w_{3}-2 j\right)+\tau}, & \bar{\xi}_{n_{\mu}, \bar{j}}=z_{r\left(n_{\mu}-w_{4}-2 \bar{j}\right)+\tau} .
\end{array}
$$

Therefore, from (3.47), (3.52), (3.53), (3.54), and (3.56), it follows that

$$
\lim _{\mu \rightarrow \infty} y_{r\left(\kappa n_{\mu}+\kappa \sigma_{\kappa}\right)+\tau}=B+\frac{C L_{\tau}}{m_{\tau}}=L_{\tau}
$$

Using the same argument to prove (3.58) and using (3.19), we can easily prove that for $i=1,2, \ldots, k, j=1,2, \ldots, m$,

$$
\lim _{\mu \rightarrow \infty} y_{r\left(\kappa n_{\mu}+\kappa \sigma_{\kappa}-r_{i}\right)+\tau}=L_{\tau}, \quad \lim _{\mu \rightarrow \infty} z_{r\left(\kappa n_{\mu}+\kappa \sigma_{\kappa}-s_{j}\right)+\tau}=m_{\tau} .
$$

Therefore, if $\delta=\epsilon\left(m_{\tau}-C\right) /\left(L_{\tau}-\epsilon-B\right)$, then in view of (3.19), (3.47), (3.58), and (3.59), there exists a $\mu_{0} \in\{1,2, \ldots\}$ such that for $j=1,2, \ldots, m$,

$$
z_{r\left(\kappa n_{\mu_{0}}+2 \phi+\kappa-s_{j}\right)+\tau} \leq C+\frac{B\left(m_{\tau}+\delta\right)}{L_{\tau}-\epsilon}=m_{\tau}+\delta
$$

and so from (3.19), (3.47), (3.48), (3.58), (3.59), and (3.60), we get

$$
y_{r\left(\kappa n_{\mu_{0}}+2 \phi+\kappa\right)+\tau} \geq B+\frac{C\left(L_{\tau}-\epsilon\right)}{m_{\tau}+\delta}=L_{\tau}-\epsilon>l_{\tau} .
$$

Using (3.19), (3.47), (3.48), (3.58), (3.59), and (3.61) and working inductively, we can easily prove that

$$
y_{r\left(\kappa n_{\mu_{0}}+2 \phi+\kappa \omega\right)+\tau} \geq L_{\tau}-\epsilon>l_{\tau}, \quad \omega=2,3, \ldots
$$

which is a contradiction since $\liminf _{n \rightarrow \infty} y_{\kappa r n+\tau}=l_{\tau}$. Therefore, since $\tau$ is an arbitrary number such that $\tau \in\{0,1, \ldots, \kappa r-1\}$, relations (3.45) are satisfied.

Moreover, from (3.19) and (3.47), we have that

$$
\lim _{n \rightarrow \infty} z_{\kappa n r+i}=\xi_{i}, \quad i=0,1, \ldots, \kappa r-1 .
$$

This completes the proof of the lemma.

In the next proposition, we study the periodicity of the positive solutions of (1.1).

Proposition 3.7. Consider (1.1), where $k, m \in\{1,2, \ldots\}, A, c_{i}, d_{j}, i \in\{1,2, \ldots, k\}, j \in$ $\{1,2, \ldots, m\}$, are positive fuzzy numbers, and $p_{i}, i \in\{1,2, \ldots, k\}, q_{j}, j \in\{1,2, \ldots, m\}$, are positive integers. If (3.10) holds and $r$ is a common divisor of the integers $p_{i}+1, q_{j}+1$, $i=1,2, \ldots, k, j=1,2, \ldots, m$, then (1.1) has periodic solutions of prime period $r$. Moreover, if $r_{i}, i=1,2, \ldots, k$, (resp., $\left.s_{j}, j=1,2, \ldots, m\right)-r_{i}, s_{j}$ are defined in (3.36)—are even (resp., odd) integers, then (1.1) has periodic solutions of prime period $2 r$. 
Proof. From (3.10), we have that $A, c_{i}, i=1,2, \ldots, k, d_{j}, j=1,2, \ldots, m$, are positive real numbers such that (3.12) and (3.13) hold. We consider functions $L_{i, a}, R_{i, a}, i=-\pi,-\pi+$ $1, \ldots, 0$, such that for $\lambda=0,1, \ldots, \phi-1, \theta=1,2, \ldots, r$, and $a \in(0,1]$,

$$
L_{-r \phi+r \lambda+\theta, a}=L_{-r+\theta, a}, \quad R_{-r \phi+r \lambda+\theta, a}=R_{-r+\theta, a},
$$

the functions $L_{w, a}, w=-r+1,-r+2, \ldots, 0$, are increasing, left continuous, and for all $w=-r+1,-r+2, \ldots, 0$, we have

$$
A+\epsilon<L_{w, a}<2 A, \quad R_{w, a}=\frac{A L_{w, a}}{L_{w, a}-A}
$$

where $\epsilon$ is a positive number such that $\epsilon<A$. Using (3.65) and since the functions $L_{w, a}$, $w=-r+1,-r+2, \ldots, 0$, are increasing, if $a_{1}, a_{2} \in(0,1], a_{1} \leq a_{2}$, we get

$$
A L_{w, a_{1}} L_{w, a_{2}}-A^{2} L_{w, a_{1}} \geq A L_{w, a_{1}} L_{w, a_{2}}-A^{2} L_{w, a_{2}}
$$

which implies that $R_{w, a}, w=-r+1,-r+2, \ldots, 0$, are decreasing functions. Moreover, from (3.65), we get

$$
L_{w, a} \leq R_{w, a}, \quad A+\epsilon \leq L_{w, a}, R_{w, a} \leq \frac{2 A^{2}}{\epsilon}
$$

and so from [18, Theorem 2.1], $\left(L_{w, a}, R_{w, a}\right), w=-r+1,-r+2, \ldots, 0$, determine the fuzzy numbers $x_{w}, w=-r+1,-r+2, \ldots, 0$, such that $\left[x_{w}\right]_{a}=\left[L_{w, a}, R_{w, a}\right], w=-r+1,-r+$ $2, \ldots, 0$. Let $x_{n}$ be a positive solution of (1.1) which satisfies (2.14) and let the initial values be positive fuzzy numbers such that (3.4) hold and the functions $L_{i, a}, R_{i, a}, i=-\pi,-\pi+$ $1, \ldots, 0, a \in(0,1]$, are defined in (3.64), (3.65); $L_{i, a}, i=-\pi,-\pi+1, \ldots, 0, a \in(0,1]$, are increasing and left continuous. Then from [16, Proposition 2], we have that for any $a \in$ $(0,1]$, the system given by (3.7), (3.12), and (3.13) has periodic solutions of prime period $r$, which means that there exists solution $\left(L_{n, a}, R_{n, a}\right), a \in(0,1]$, of the system such that

$$
L_{n+r, a}=L_{n, a}, \quad R_{n+r, a}=R_{n, a}, \quad a \in(0,1] .
$$

Therefore, from (2.22) and (3.68), we have that (1.1) has periodic solutions of prime period $r$.

Now, suppose that $r_{i}, i=1,2, \ldots, k$, (resp., $s_{i}, j=1,2, \ldots, m$ ) are even (resp., odd) integers. We consider the functions $L_{i, a}, R_{i, a}, i=-\pi,-\pi+1, \ldots, 0$, such that analogous relations (3.37), (3.38), and (3.43) hold, $L_{w, a}, w=-r+1, \ldots, 0$, are increasing, left continuous functions, and the first relation of (3.65) holds. Arguing as above, the solution $x_{n}$ of (1.1) with initial values $x_{i}, i=-\pi,-\pi+1, \ldots, 0$, satisfying (3.4), where $L_{i, a}, R_{i, a}$, $i=-\pi,-\pi+1, \ldots, 0$, are defined above, is a periodic solution of prime period $2 r$.

In the following proposition, we study the convergence of the positive solutions of (1.1). 
Proposition 3.8. Consider (1.1), where $k, m \in\{1,2, \ldots\}, A, c_{i}, d_{j}, i \in\{1,2, \ldots, k\}, j \in$ $\{1,2, \ldots, m\}$, are positive fuzzy numbers, and $p_{i}, i \in\{1,2, \ldots, k\}, q_{j}, j \in\{1,2, \ldots, m\}$, are positive integers. Then the following statements are true.

(i) If (3.11), holds, then (1.1) has a unique positive equilibrium $x$ and every positive solution of (1.1) nearly converges to the unique positive equilibrium $x$ with respect to $D$ as $n \rightarrow \infty$ and converges to $x$ with respect to $D_{1}$ as $n \rightarrow \infty$.

(ii) If (3.10) is satisfied and $r$ is the greatest common divisor of the integers $p_{i}+1, q_{j}+1$, $i=1,2, \ldots, k, j=1,2, \ldots, m$, such that (3.36) holds, then every positive solution of (1.1) nearly converges to a period $\kappa r$ solution of (1.1) with respect to $D$ as $n \rightarrow \infty$ and converges to a period $\kappa r$ solution of (1.1) with respect to $D_{1}$ as $n \rightarrow \infty ; \kappa$ is defined in (3.44).

Proof. (i) Let $x_{n}$ be a positive solution of (1.1) which satisfies (2.14). Since (3.7) and (3.11) hold, we can apply [16, Proposition 4] and we have that for any $a \in(0,1]$, there exist the $\lim _{n \rightarrow \infty} L_{n, a}, \lim _{n \rightarrow \infty} R_{n, a}$, and

$$
\lim _{n \rightarrow \infty} L_{n, a}=L_{a}, \quad \lim _{n \rightarrow \infty} R_{n, a}=R_{a}, \quad a \in(0,1]
$$

where

$$
\begin{aligned}
& L_{a}=\frac{A_{l, a} A_{r, a}-C_{a} D_{a}}{A_{r, a}-C_{a}}, \quad R_{a}=\frac{A_{l, a} A_{r, a}-C_{a} D_{a}}{A_{l, a}-D_{a}}, \\
& C_{a}=\frac{\sum_{i=1}^{k} c_{i, l, a}}{\sum_{j=1}^{m} d_{j, r, a}}, \quad D_{a}=\frac{\sum_{i=1}^{k} c_{i, r, a}}{\sum_{j=1}^{m} d_{j, l, a}} .
\end{aligned}
$$

In addition, from (3.3) and (3.70), we get

$$
L_{a} \geq \frac{B^{2}-Z^{2}}{C-W}=\lambda, \quad R_{a} \leq \frac{C^{2}-W^{2}}{B-Z}=\mu,
$$

where $B, C$ (resp., $Z, W$ ) are defined in (3.3) (resp., (3.5)). Then from (3.69), (3.71), and arguing as in $[13,14,15]$, we can easily prove that $L_{a}, R_{a}$ determine a fuzzy number $x$ such that $[x]_{a}=\left[L_{a}, R_{a}\right]$. Finally, using (3.70), we take that $x$ is the unique positive equilibrium of (1.1). Using relations (3.11), (3.69), and arguing as in [15, Proposition 2], we can prove that every positive solution of (1.1) nearly converges to the unique positive equilibrium $x$ with respect to $D$ as $n \rightarrow \infty$ and converges to $x$ with respect to $D_{1}$ as $n \rightarrow \infty$.

(ii) Suppose that (3.10) holds. Let $x_{n}$ be a positive solution of (1.1) such that (2.14) holds. Since $\left(L_{n, a}, R_{n, a}\right)$ is a positive solution of the system which is defined by (3.7), (3.12), and (3.13), from Lemma 3.6, we have that

$$
\lim _{n \rightarrow \infty} L_{\kappa n r+l, a}=\epsilon_{l, a}, \quad \lim _{n \rightarrow \infty} R_{\kappa n r+l, a}=\xi_{l, a}, \quad a \in(0,1], l=0,1, \ldots, \kappa r-1,
$$

where $\kappa$ is defined in (3.44). Using (3.72) and arguing as in [15, Proposition 2], we can prove that every positive solution of (1.1) nearly converges to a period $\kappa r$ solution of (1.1) with respect to $D$ as $n \rightarrow \infty$ and converges to a period $\kappa r$ solution of (1.1) with respect to $D_{1}$ as $n \rightarrow \infty$. Thus, the proof of the proposition is completed.

From Propositions 3.2-3.8, it is obvious that (1.1) exhibits the trichotomy character described concentratively by the following proposition. 
Proposition 3.9. Consider the fuzzy difference equation (1.1), where $k, m \in\{1,2, \ldots\}$, and $A, c_{i}, d_{j}, i \in\{1,2, \ldots, k\}, j \in\{1,2, \ldots, m\}$, are positive fuzzy numbers. Then (1.1) possesses the following trichotomy.

(i) If relation (3.1) is satisfied, then (1.1) has unbounded solutions.

(ii) If (3.10) holds and $r$ is the greatest common divisor of the integers $p_{i}+1, q_{j}+1$, $i=1,2, \ldots, k, j=1,2, \ldots, m$, such that (3.36) holds, then every positive solution of (1.1) nearly converges to a period $\kappa r$ solution of (1.1) with respect to $D$ as $n \rightarrow \infty$ and converges to a period $\kappa r$ solution of (1.1) with respect to $D_{1}$ as $n \rightarrow \infty$.

(iii) If (3.11) holds, then every positive solution of (1.1) nearly converges to the unique positive equilibrium $x$ with respect to $D$ as $n \rightarrow \infty$ and converges to $x$ with respect to $D_{1}$ as $n \rightarrow \infty$.

In the next proposition, we study the asymptotic stability of the unique positive equilibrium of (1.1).

Proposition 3.10. Consider the fuzzy difference equation (1.1), where $k, m \in\{1,2, \ldots\}$, $A, c_{i}, d_{j}, i \in\{1,2, \ldots, k\}, j \in\{1,2, \ldots, m\}$, are positive fuzzy numbers, and $p_{i}, i \in\{1,2, \ldots, k\}$, $q_{j}, j \in\{1,2, \ldots, m\}$, are positive integers such that (3.11) holds. Suppose that there exists a positive number $\theta$ such that

$$
\theta<B, \quad Z<\frac{2 B+C-\theta-\sqrt{(C-\theta)^{2}+4 B C}}{2},
$$

where $B, C$ are defined in (3.3) and $Z$ is defined in (3.5). Then the unique positive equilibrium $x$ of (1.1) is nearly asymptotically stable.

Proof. Since (3.11) holds, from Proposition 3.8, equation (1.1) has a unique positive equilibrium $x$ which satisfies (2.15).

Let $\epsilon$ be a positive real number. Since (3.18) holds, we can define the positive real number $\delta$ as follows:

$$
\delta<\min \{\epsilon, \lambda, \theta, B-Z\}
$$

Let $x_{n}$ be a positive solution of (1.1) such that

$$
D\left(x_{-i}, x\right) \leq \delta \leq \epsilon, \quad i=0,1, \ldots, \pi .
$$

From (3.75), we have

$$
\left|L_{-i, a}-L_{a}\right| \leq \delta, \quad\left|R_{-i, a}-R_{a}\right| \leq \delta, \quad i=0,1, \ldots, \pi, a \in(0,1] .
$$

In addition, from (3.3), (3.7), (3.74), and (3.76) and since $\left(L_{a}, R_{a}\right)$ satisfies (3.7), we get

$$
\begin{aligned}
L_{1, a}-L_{a} & =A_{l, a}+\frac{\sum_{i=1}^{k} c_{i, l, a} L_{-p_{i}, a}}{\sum_{j=1}^{m} d_{j, r, a} R_{-q_{j}, a}}-L_{a} \leq A_{l, a}+\frac{\sum_{i=1}^{k} c_{i, l, a}\left(L_{a}+\delta\right)}{\sum_{j=1}^{m} d_{j, r, a}\left(R_{a}-\delta\right)}-L_{a} \\
& =\delta \frac{C_{a}-A_{l, a}+L_{a}}{R_{a}-\delta} \leq \delta \frac{R_{a}-(B-Z)}{R_{a}-\delta} .
\end{aligned}
$$


From (3.74) and (3.77), it is obvious that

$$
\left|L_{1, a}-L_{a}\right|<\delta<\epsilon
$$

Moreover, arguing as above, we can easily prove that

$$
R_{1, a}-R_{a} \leq \delta \frac{D_{a}-A_{r, a}+R_{a}}{L_{a}-\delta}
$$

We claim that

$$
\theta<L_{a}-R_{a}+A_{r, a}-D_{a}, \quad a \in(0,1]
$$

We fix an $a \in(0,1]$ and we concider the function

$$
g(h)=\frac{A_{l, a} A_{r, a}-D_{a} h}{A_{r, a}-h}-\frac{A_{l, a} A_{r, a}-D_{a} h}{A_{l, a}-D_{a}}+A_{r, a}-D_{a},
$$

where $h$ is a nonnegative real variable. Moreover, we consider the function

$$
f(x, y, z)=\frac{x^{2}-(2 x+y) z+z^{2}}{x-z}-\theta
$$

where $B \leq x \leq y \leq C$ and $W \leq z \leq Z, B, C$ (resp., $W, Z$ ) are defined in (3.3) (resp., (3.5)). Using (3.82), we can easily prove that the function $f$ is increasing (resp., decreasing) (resp., decreasing) with respect to $x$ (resp., $y$ ) (resp., $z$ ) for all $y, z$ (resp., $x, z)$ (resp., $x, y$ ) and so from (3.73),

$$
f(x, y, z)>f(B, C, Z)=\frac{B^{2}-(2 B+C) Z+Z^{2}}{B-Z}-\theta>0 .
$$

Therefore, from (3.3), (3.81), (3.82), and (3.83), we have

$$
g(0)=f\left(A_{l, a}, A_{r, a}, D_{a}\right)+\theta>\theta
$$

In addition, from (3.81), we can prove that $g$ is an increasing function with respect to $h$ and so we have $g(0)<g\left(C_{a}\right), a \in(0,1]$. Therefore, from (3.70), (3.81), and (3.84), relation (3.80) is true. Hence, from (3.74), (3.79), and (3.80), we get

$$
\left|R_{1, a}-R_{a}\right|<\delta<\epsilon
$$

From (3.7), (3.76), (3.78), and (3.85) and working inductively, we can easily prove that

$$
\left|L_{n, a}-L_{a}\right| \leq \epsilon, \quad\left|R_{n, a}-R_{a}\right| \leq \epsilon, \quad a \in(0,1], n=0,1, \ldots
$$


and so

$$
D\left(x_{n}, x\right) \leq \epsilon, \quad n \geq 0 .
$$

Therefore, the positive equilibrium $x$ is stable. Moreover, from Proposition 3.8, we have that every positive solution of (1.1) nearly tends to $x$ with respect to $D$ as $n \rightarrow \infty$. So, $x$ is nearly asymptotically stable. So, the proof of the proposition is completed.

Finally, we study the oscillatory behavior of the positive solutions of the fuzzy difference equation

$$
x_{n+1}=A+\frac{\sum_{s=0}^{k} c_{2 s+1} x_{n-2 s-1}}{\sum_{s=0}^{k} d_{2 s+2} x_{n-2 s}},
$$

where $k$ is a positive integer, and $A, c_{2 s+1}, d_{2 s+2}, s \in\{0,1, \ldots, k\}$, are positive fuzzy numbers. Obviously, (3.88) is a special case of (1.1).

In what follows, we need to study the oscillatory behavior of the positive solutions of the system of ordinary difference equations

$$
\begin{aligned}
& y_{n+1}=B+\frac{\sum_{s=0}^{k} a_{2 s+1} y_{n-2 s-1}}{\sum_{s=0}^{k} b_{2 s+2} z_{n-2 s}}, \quad n=0,1, \ldots, \\
& z_{n+1}=C+\frac{\sum_{s=0}^{k} e_{2 s+1} z_{n-2 s-1}}{\sum_{s=0}^{k} h_{2 s+2} y_{n-2 s}},
\end{aligned}
$$

where $k$ is a positive integer, $B, C, a_{2 s+1}, b_{2 s+2}, e_{2 s+1}, h_{2 s+2}, s \in\{0,1, \ldots, k\}$, are positive real constants, and the initial values $y_{j}, z_{j}, j=-2 k-1,-2 k, \ldots, 0$, are positive real numbers.

Let $\left(y_{n}, z_{n}\right)$ be a positive solution of (3.89). We say that the solution $\left(y_{n}, z_{n}\right)$ oscillates about $(y, z), y, z \in \mathbb{R}^{+}$, if for every $n_{0} \in \mathbb{N}$, there exist $s, m \in \mathbb{N}, s, m \geq n_{0}$, such that

$$
\begin{array}{rlrl}
\left(y_{s}-y\right)\left(y_{m}-y\right) & \leq 0, & & \left(z_{s}-z\right)\left(z_{m}-z\right) \leq 0 \\
\left(y_{s}-y\right)\left(z_{s}-z\right) \geq 0, & & \left(y_{m}-y\right)\left(z_{m}-z\right) \geq 0 .
\end{array}
$$

Lemma 3.11. Consider system (3.89), where $k$ is a positive integer, $B, C, a_{2 s+1}, b_{2 s+2}, e_{2 s+1}$, $h_{2 s+2}, s \in\{0,1, \ldots, k\}$, are positive real constants, and the initial values $y_{j}, z_{j}, j=-2 k-$ $1,-2 k, \ldots, 0$, are positive real numbers. A positive solution $\left(y_{n}, z_{n}\right)$ of system (3.89) oscillates about the unique positive equilibrium $(\bar{x}, \bar{y})$ of system (3.89) if either the relations

$$
\Lambda \geq \max \left\{\Lambda_{1, s}, \Lambda_{2, s}\right\}, \quad \Delta \geq \max \left\{\Delta_{1, s}, \Delta_{2, s}\right\}, \quad s=0,1, \ldots, k
$$

or the relations

$$
\Lambda \leq \min \left\{\Lambda_{1, s}, \Lambda_{2, s}\right\}, \quad \Delta \leq \min \left\{\Delta_{1, s}, \Delta_{2, s}\right\}, \quad s=0,1, \ldots, k
$$


hold, where for $s=0,1, \ldots, k$,

$$
\begin{gathered}
\Lambda=\frac{\sum_{s=0}^{k} e_{2 s+1} z_{-2 s-1}}{\sum_{s=0}^{k} h_{2 s+2} y_{-2 s}}, \quad \Delta=\frac{\sum_{s=0}^{k} a_{2 s+1} y_{-2 s-1}}{\sum_{s=0}^{k} b_{2 s+2} z_{-2 s}}, \\
\Delta_{1, s}=\frac{1}{a_{2 s+1}}\left[\mu \frac{\bar{y}}{\bar{z}}\left(\sum_{j=0}^{s} b_{2 j+2} \bar{z}+\sum_{j=s+1}^{k} b_{2 j+2} z_{-2 j+2+2 s}\right)-\left(\sum_{j=0}^{s-1} a_{2 j+1} \bar{y}+\sum_{j=s+1}^{k} a_{2 j+1} y_{-2 j+1+2 s}\right)\right]-B, \\
\Delta_{2, s}=\frac{1}{h_{2 s+2}}\left[\frac{\bar{y}}{\lambda \bar{z}}\left(\sum_{j=0}^{s-1} e_{2 j+1} \bar{z}+\sum_{j=s}^{k} e_{2 j+1} z_{-2 j+2 s}\right)-\left(\sum_{j=0}^{s-1} h_{2 j+2} \bar{y}+\sum_{j=s+1}^{k} h_{2 j+2} y_{-2 j+1+2 s}\right)\right]-B, \\
\Lambda_{1, s}=\frac{1}{e_{2 s+1}}\left[\lambda \frac{\bar{z}}{\bar{y}}\left(\sum_{j=0}^{s} h_{2 j+2} \bar{y}+\sum_{j=s+1}^{k} h_{2 j+2} y_{-2 j+2+2 s}\right)-\left(\sum_{j=0}^{s-1} e_{2 j+1} \bar{z}+\sum_{j=s+1}^{k} e_{2 j+1} z_{-2 j+1+2 s}\right)\right]-C, \\
\Lambda_{2, s}=\frac{1}{b_{2 s+2}}\left[\frac{\bar{z}}{\mu \bar{y}}\left(\sum_{j=0}^{s-1} a_{2 j+1} \bar{y}+\sum_{j=s}^{k} a_{2 j+1} y_{-2 j+2 s}\right)-\left(\sum_{j=0}^{s-1} b_{2 j+2} \bar{z}+\sum_{j=s+1}^{k} b_{2 j+2} z_{-2 j+1+2 s}\right)\right]-C, \\
\lambda=\frac{\sum_{s=0}^{k} e_{2 s+1},}{\sum_{s=0}^{k} h_{2 s+2}} \quad \mu=\frac{\sum_{s=0}^{k} a_{2 s+1}}{\sum_{s=0}^{k} b_{2 s+2}}
\end{gathered}
$$

Proof. Suppose that (3.91) hold. We prove that for $\rho=0,1, \ldots, k$,

$$
y_{2 \rho+1} \geq \bar{y}, \quad z_{2 \rho+1} \geq \bar{z}, \quad y_{2 \rho+2} \leq \bar{y}, \quad z_{2 \rho+2} \leq \bar{z} .
$$

From (3.89) and (3.91), we have

$$
\begin{gathered}
y_{1}=B+\frac{\sum_{s=0}^{k} a_{2 s+1} y_{-2 s-1}}{\sum_{s=0}^{k} b_{2 s+2} z_{-2 s}}=B+\Delta \geq B+\Delta_{1, k}=\bar{y}, \\
z_{1}=C+\Lambda \geq C+\Lambda_{1, k}=\bar{z} .
\end{gathered}
$$

Since from (3.91), $\Lambda \geq \Lambda_{2,0}$ and $\Delta \geq \Delta_{2,0}$, then from (3.89), we have

$$
\begin{gathered}
y_{2}=B+\frac{\sum_{s=0}^{k} a_{2 s+1} y_{-2 s}}{b_{2} z_{1}+\sum_{s=1}^{k} b_{2 s+2} z_{1-2 s}} \leq B+\frac{(C+\Lambda) b_{2}+\sum_{s=1}^{k} b_{2 s+2} z_{1-2 s}}{b_{2} z_{1}+\sum_{s=1}^{k} b_{2 s+2} z_{1-2 s}} \frac{\mu \bar{y}}{\bar{z}}=B+\frac{\mu \bar{y}}{\bar{z}}=\bar{y}, \\
z_{2} \leq C+\frac{\lambda \bar{z}}{\bar{y}}=\bar{z} .
\end{gathered}
$$

Using (3.89), (3.91), (3.95), and (3.96), relations $\Delta \geq \Delta_{1, \rho-1}, \Lambda \geq \Lambda_{1, \rho-1}$ (resp., $\Delta \geq$ $\left.\Delta_{2, \rho}, \Lambda \geq \Lambda_{2, \rho}\right), \rho=1,2, \ldots, k$, and working inductively, we can easily prove (3.94) for $\rho=$ $1,2, \ldots, k$ :

$$
y_{2 \rho+1} \geq \bar{y}, \quad z_{2 \rho+1} \geq \bar{z} \quad\left(\text { resp., } y_{2 \rho+2} \leq \bar{y}, z_{2 \rho+2} \leq \bar{z}\right) .
$$


Therefore, (3.94) hold for $\rho=0,1, \ldots, k$. Then since (3.94) hold for $\rho=0,1, \ldots, k$, using (3.89) and working inductively, we can easily prove that(3.94) hold for any $\rho=k+1, k+$ $2, \ldots$, and so if (3.91) hold, the proof of the lemma is completed.

Similarly, if (3.92) are satisfied, then we can easily prove that

$$
y_{2 \rho+1} \leq \bar{y}, \quad z_{2 \rho+1} \leq \bar{z}, \quad y_{2 \rho+2} \geq \bar{y}, \quad z_{2 \rho+2} \geq \bar{z}, \quad \rho=0,1, \ldots
$$

This completes the proof of the lemma.

Using Lemma 3.11 and arguing as in [13, Proposition 2.4], we can easily prove the following proposition which concerns the oscillatory behavior of the positive solutions of the fuzzy difference equation (3.88).

Proposition 3.12. Consider (3.88), where $k$ is a positive integer, and $A, c_{2 s+1}, d_{2 s+2}, s \in\{0$, $1, \ldots, k\}$, are positive fuzzy numbers. Then a positive solution $x_{n}$ of (3.88) satisfying (2.14) oscillates about the positive equilibrium $x$, which satisfies (2.15) if, for any $s=0,1, \ldots, k$ and $a \in(0,1]$, either the relations

$$
\bar{\Lambda}_{a} \geq \max \left\{\bar{\Lambda}_{1, s, a}, \bar{\Lambda}_{2, s, a}\right\}, \quad \bar{\Delta}_{a} \geq \max \left\{\bar{\Delta}_{1, s, a}, \bar{\Delta}_{2, s, a}\right\}
$$

or the relations

$$
\bar{\Lambda}_{a} \leq \min \left\{\bar{\Lambda}_{1, s, a}, \bar{\Lambda}_{2, s, a}\right\}, \quad \bar{\Delta}_{a} \leq \min \left\{\bar{\Delta}_{1, s, a}, \bar{\Delta}_{2, s, a}\right\}
$$

hold, where $\bar{\Lambda}_{a}, \bar{\Delta}_{a}, \bar{\Lambda}_{1, s, a}, \bar{\Lambda}_{2, s, a}, \bar{\Delta}_{1, s, a}, \bar{\Delta}_{2, s, a}$ are defined for the analogous system (3.7) in the same way as $\Lambda, \Delta, \Lambda_{1, s}, \Lambda_{2, s}, \Delta_{1, s}, \Delta_{2, s}$ were defined in Lemma 3.11 for system (3.89).

Using Proposition 3.12, we take the following corollary.

Corollary 3.13. Consider (3.88), where $k$ is a positive integer, and $A, c_{2 s+1}, d_{2 s+2}, s \in$ $\{0,1, \ldots, k\}$, are positive fuzzy numbers. Then a positive solution $x_{n}$ of (3.88) satisfying (2.14) oscillates about the positive equilibrium $x$, which satisfies (2.15) if, for any $p=0,1, \ldots, k$ and $a \in(0,1]$, either the relations

$$
\begin{aligned}
& L_{-2 k-1+2 p, a} \geq L_{a}, \quad R_{-2 k-1+2 p, a} \geq R_{a}, \\
& L_{-2 k+2 p, a} \leq L_{a}, \quad R_{-2 k+2 p, a} \leq R_{a}
\end{aligned}
$$

or the relations

$$
\begin{aligned}
& L_{-2 k-1+2 p, a} \leq L_{a}, \quad R_{-2 k-1+2 p, a} \leq R_{a}, \\
& L_{-2 k+2 p, a} \geq L_{a}, \quad R_{-2 k+2 p, a} \geq R_{a}
\end{aligned}
$$

hold.

\section{Acknowledgment}

This work is a part of the first author Doctoral thesis. 


\section{References}

[1] R. P. Agarwal, Difference Equations and Inequalities, Monographs and Textbooks in Pure and Applied Mathematics, vol. 155, Marcel Dekker, New York, 1992.

[2] D. Benest and C. Froeschle, Analysis and Modelling of Discrete Dynamical Systems, Advances in Discrete Mathematics and Applications, vol. 1, Gordon and Breach Science Publishers, Amsterdam, 1998.

[3] E. Y. Deeba and A. De Korvin, Analysis by fuzzy difference equations of a model of $\mathrm{CO}_{2}$ level in the blood, Appl. Math. Lett. 12 (1999), no. 3, 33-40.

[4] E. Y. Deeba, A. De Korvin, and E. L. Koh, A fuzzy difference equation with an application, J. Differ. Equations Appl. 2 (1996), no. 4, 365-374.

[5] R. DeVault, G. Ladas, and S. W. Schultz, On the recursive sequence $x_{n+1}=A / x_{n}^{p}+B / x_{n-1}^{q}$, Advances in Difference Equations (Veszprém, 1995), Gordon and Breach, Amsterdam, 1997, pp. 125-136.

[6] On the recursive sequence $x_{n+1}=A / x_{n}+1 / x_{n-2}$, Proc. Amer. Math. Soc. 126 (1998), no. 11, 3257-3261.

[7] L. Edelstein-Keshet, Mathematical Models in Biology, The Random House/Birkhäuser Mathematics Series, Random House, New York, 1988.

[8] S. N. Elaydi, An Introduction to Difference Equations, Undergraduate Texts in Mathematics, Springer-Verlag, New York, 1996.

[9] S. Heilpern, Fuzzy mappings and fixed point theorem, J. Math. Anal. Appl. 83 (1981), no. 2, $566-569$.

[10] G. J. Klir and B. Yuan, Fuzzy Sets and Fuzzy Logic: Theory and Applications, Prentice Hall PTR, New Jersey, 1995.

[11] V. L. Kocić and G. Ladas, Global Behavior of Nonlinear Difference Equations of Higher Order with Applications, Mathematics and its Applications, vol. 256, Kluwer Academic Publishers Group, Dordrecht, 1993.

[12] H. T. Nguyen and E. A. Walker, A First Course in Fuzzy Logic, CRC Press, Florida, 1997.

[13] G. Papaschinopoulos and B. K. Papadopoulos, On the fuzzy difference equation $x_{n+1}=A+B / x_{n}$, Soft Comput. 6 (2002), 436-440.

[14] On the fuzzy difference equation $x_{n+1}=A+x_{n} / x_{n-m}$, Fuzzy Sets and Systems 129 (2002), no. 1, 73-81.

[15] G. Papaschinopoulos and G. Stefanidou, Boundedness and asymptotic behavior of the solutions of a fuzzy difference equation, Fuzzy Sets and Systems 140 (2003), no. 3, 523-539.

[16] Trichotomy of a system of two difference equations, J. Math. Anal. Appl. 289 (2004), no. 1, 216-230.

[17] M. L. Puri and D. A. Ralescu, Differentials of fuzzy functions, J. Math. Anal. Appl. 91 (1983), no. 2, 552-558.

[18] C. Wu and B. Zhang, Embedding problem of noncompact fuzzy number space $E^{\sim}$ (I), Fuzzy Sets and Systems 105 (1999), no. 1, 165-169.

G. Stefanidou: Department of Electrical and Computer Engineering, Democritus University of Thrace, 67100 Xanthi, Greece

E-mail address: tfele@yahoo.gr

G. Papaschinopoulos: Department of Electrical and Computer Engineering, Democritus University of Thrace, 67100 Xanthi, Greece

E-mail address: gpapas@ee.duth.gr 